\title{
Percepción de enfermería. Una mirada actual a la cultura de seguridad en el quirófano*
}

\author{
Nursing perception. A current look at the safety culture in the operating room \\ Percepção da enfermagem. Um olhar atual à cultura de segurança no quirófano
}

Liset Pérez Bolaños ${ }^{\text {a }}$
Centro de Investigaciones Médico Quirúrgicas, Cuba
lisetpb@infomed.sld.cu
ORCID: https://orcid.org/0000-0002-7589-7882
DOI: https://doi.org/10.11144/Javeriana.ie22.pema

Recibido: 28 Noviembre 2018

Aceptado: 12 Diciembre 2019

Publicado: 22 Julio 2020

\section{Resumen:}

Introducción: La cultura de seguridad del paciente se considera una prioridad en la asistencia sanitaria para la disminución de los eventos adversos en los quirófanos donde su ocurrencia no se informa y ello genera riesgos. Objetivo: Caracterizar la cultura de seguridad del paciente desde la percepción de los profesionales de enfermería de quirófano. Método: Estudio descriptivo transversal que recogió los resultados de la encuesta anónima de cultura de seguridad del paciente elaborada por la Agencia para la Investigación y Calidad en Salud, adaptada al español en el curso poscongreso en Electrocirugías para Instrumentistas, realizado a una muestra de 67 profesionales de enfermería, trabajadores de 10 quirófanos, localizados en La Habana (Cuba). Se evaluó la percepción que tienen los profesionales de enfermería. Se analizaron las respuestas positivas y negativas a los 50 ítems, incluyendo la calificación global de seguridad. El procesamiento de la información de la encuesta se codificó y se colectó en una base de datos confeccionada en el procesador estadístico Excel y luego, para su análisis, se convirtió a SPSS versión 21.0 para Windows. Resultado: Se analizaron 67 encuestas; el 100 \% de los encuestados calificó de positivo el grado global de seguridad. Como fortaleza se destacan las dimensiones: aprendizaje organizacional, apoyo de la dirección y trabajo en equipo entre unidades. El resto de las dimensiones, excepto la respuesta no punitiva (clasificada como debilidad), se clasificaron como oportunidades de mejora. Conclusiones: La cultura de seguridad del paciente debe progresar de la culpabilidad al aprendizaje.

Palabras clave: seguridad del paciente, quirófano, percepción, gestión de la seguridad.

\section{Abstract:}

Introduction: The culture of patient safety is considered a priority in healthcare settings to reduce the incidence of adverse events in operating rooms, where their occurrence is not reported, thus leading to risks. Objective: To describe the culture of patient safety from the perception of operating room nursing professionals. Method: Cross-sectional descriptive study that collected the results of the anonymous patient safety culture survey prepared by the Agency for Health Research and Quality, adapted to Spanish in the post-congress course on Electrosurgery for Instrumentalists. The survey was conducted on a sample of 67 professional nurses who work at 10 operating rooms located in Havana (Cuba) to evaluate their perception. Positive and negative responses to the 50 items were analyzed, including an overall safety rating. Survey information was coded, collected, and processed in a database compiled in the Excel statistical processor, which was then converted for analysis to SPSS version 21.0 for Windows. Result: Of the 67 surveys analyzed, $100 \%$ of the respondents rated the overall degree of safety as positive. The dimensions organizational learning, management support, and teamwork between units were highlighted as strengths. The rest of the dimensions (except for the nonpunitive response dimension, which was classified as a weakness) were classified as opportunities for improvement. Conclusions: The culture of patient safety must progress from guilt to learning.

Keywords: patient safety, operating room, perception, safety management.

\section{Resumo:}

Introdução: A cultura de segurança do paciente é considerada prioridade na assistência sanitária para a diminuição dos eventos adversos nos quirófanos onde a ocorrência não é relatada e isso gera risco. Objetivo: Caracterizar a cultura de segurança do paciente desde a percepção dos profissionais de enfermagem de quirófano. Método:Estudo descritivo transversal que recolheu resultados do inquérito anónimo de cultura de segurança do paciente elaborado pela Agência para a Pesquisa e Qualidade em Saúde adaptada ao espanhol no curso pós-congresso em Eletrocirurgias para Instrumentistas, realizado a amostra de 67 profissionais de

Notas de autor

\footnotetext{
a Autora de correspondencia. Correo electrónico: lisetpb@infomed.sld.cu
} 
enfermagem, trabalhadores de dez quirófanos, localizados em Havana (Cuba). A percepção dos profissionais de enfermagem foi avaliada. Analisaram-se as respostas positivas e negativas a 50 itens, incluindo a qualificação global de segurança. $O$ processamento das informações da pesquisa foi codificado e coletado em um banco de dados compilado no processador estatístico Excel e em seguida, para análise, convertido para o SPSS versão 21.0 para Windows. Resultado: 67 inquéritos foram analisados; $100 \%$ dos perguntados classificou de positivo o grão geral de segurança. Como força destacam-se as dimensões: aprendizado organizacional, suporte gerencial e trabalho em equipe entre as unidades. $\mathrm{O}$ restante das dimensões, exceto a resposta não punitiva (classificada como fraqueza), foi classificado como oportunidade de melhoria. Conclusões: A cultura de segurança do paciente deve progredir da culpa para a aprendizagem.

Palavras-chave: segurança do paciente, quirófano, percepção, gestão de segurança.

\section{Introducción}

Hoy en día, la cultura sobre seguridad del paciente se considera una prioridad en cualquier sistema sanitario. Constituye una herramienta esencial para prevenir eventos adversos (1-5), pues identifica fortalezas y áreas de mejora continua. Las enfermeras desempeñan un papel importante en la seguridad de los pacientes, porque son responsables de la atención directa y continua del paciente (2), lo cual justifica nuestro estudio para saber qué aspectos debemos mejorar, a efectos de proporcionar cuidados seguros y de calidad a nuestros pacientes.

La cultura de seguridad del paciente puede definirse como el modo de pensar sobre la seguridad del paciente y la forma en que se implementa en una organización. Las organizaciones con una cultura positiva comparten la percepción de la importancia de la seguridad y confían en la eficacia de las acciones preventivas $(3,5)$.

La mayoría de los estudios que se han desarrollado plantean la necesidad de un cambio cultural de las organizaciones que permita promover las buenas prácticas de los profesionales de salud para gestionar adecuadamente la atención de salud y, con esto, garantizar la seguridad en la entrega de las prestaciones (1-9).

El principal rol de la enfermera para mejorar la seguridad es cambiar el patrón de ocultación de fallos o errores, conductas reactivas ante los eventos adversos. La meta es que el profesional asistencial se sienta cómodo trabajando en equipo sin condicionantes jerárquicos negativos y con una comunicación activa entre sí. Este nuevo clima laboral debe facilitar el desarrollo de, por ejemplo, la facultad de reconocer, comunicar, aprender de los errores, y la humildad para solicitar ayuda al resto del grupo cuando las condiciones individuales lo aconsejen. Dialogar sobre los eventos adversos con naturalidad (4) es un primer paso, necesario, para lograr una cultura de seguridad que contribuya a reducir su ocurrencia. Los conocimientos, las actitudes y las conductas profesionales en torno a los eventos adversos enmarcan esa cultura y caracterizarlas - que es nuestro objetivo de esta investigación - puede darnos pistas sobre dónde interesa concentrar los esfuerzos para disminuir la aparición de eventos adversos.

\section{Método}

Estudio descriptivo-transversal realizado en 10 quirófanos de La Habana, del 2016 a mayo del 2018. La muestra la constituyeron 67 profesionales de enfermería de quirófano. Los criterios de inclusión fueron los profesionales que se encontraban en el curso Poscongreso en Electrocirugías para Instrumentistas y que aceptaron participar en el estudio. La cantidad de individuos que se evaluó se estimó con el instrumento propuesto para calcular un coeficiente de fiabilidad (alfa de Cronbach) con un $95 \%$ de confianza y una precisión absoluta de 0,2 unidades (ancho del intervalo de confianza) (10).

Se aplicó la encuesta anónima de cultura de seguridad del paciente, elaborada por la Agencia para la Investigación y Calidad en Salud, adaptada al español (11). Se evaluó la percepción que tienen los profesionales de enfermería. Se analizaron las respuestas positivas y negativas a los 50 ítems, incluyendo la calificación global de seguridad. Cada ítem se valoró por medio de una escala tipo Likert. Adicionalmente, se incluyeron las variables clasificatorias socioprofesionales. 
Los indicadores compuestos para cada una de las dimensiones de la escala se calcularon aplicando la siguiente fórmula:

\section{$\Sigma=\frac{\text { Número de respuestas positivas en los ítems de cada dimensión }}{\text { Número de respuestas totales en los ítems de cada dimensión }}$}

Para clasificar un ítem o una dimensión como fortaleza se emplearon los siguientes criterios:

- $\quad \geq 75 \%$ de respuestas positivas ("de acuerdo" o "casi siempre/siempre") a preguntas formuladas en positivo.

- $\quad \geq 75 \%$ de respuestas negativas ("en desacuerdo" o "nunca/casi nunca") a preguntas formuladas en negativo.

Para clasificar un ítem o una dimensión como oportunidad de mejora se emplearon los siguientes criterios:

- $\quad \geq 50 \%$ de respuestas negativas ("en desacuerdo" o "nunca/casi nunca") a preguntas formuladas en positivo.

- $\quad \geq 50 \%$ de respuestas positivas ("de acuerdo" o "casi siempre/siempre") a preguntas formuladas en negativo.

Se estimaron las distribuciones de frecuencia para describir las características de los profesionales encuestados. Se evaluó la fiabilidad calculando el estadístico alfa de Cronbach. Con respecto al porcentaje de respuestas positivas para cada dimensión, se estimó el intervalo de confianza al $95 \%$ de cada una. Para el análisis estadístico se utilizó el sistema SPSS para Windows versión 21.0. Se prefijó el error de tipo I, con un alfa $=0,05$. Los resultados se muestran en tablas y figuras para su mejor comprensión.

La presente investigación cumplió con los requerimientos éticos investigativos con la Declaración de Helsinki.

\section{Resultados}

El $100 \%$ del personal encuestado corresponde a profesionales de enfermería de 10 unidades quirúrgicas de La Habana. De manera global, el 83,5\% de los encuestados lleva trabajando 5 años o más en el hospital y servicio. La mayoría $(53,7 \%)$ trabaja entre 32 y 47 horas por semana, y no es despreciable la frecuencia de los que trabajan más de 57 horas por semana (20,9\%). Destacan en la muestra encuestada aquellos profesionales con más de 20 años de experiencia $(67,2 \%)$ que, sumados a los que tienen entre 10 y 19 años de experiencia, cubren más del $90 \%$ del total de profesionales encuestados (tabla 1 ).

TABLA 1

Características del personal encuestado

\begin{tabular}{|c|c|c|c|}
\hline \multicolumn{2}{|c|}{ Características de los encuestados } & 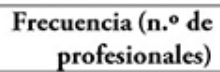 & Porcentaje \\
\hline \multirow{4}{*}{$\begin{array}{l}\text { Años de trabajo en el hospital y } \\
\text { unidad }\end{array}$} & $1-4$ & 11 & 16,4 \\
\hline & $5-9$ & 21 & 31,3 \\
\hline & $10-19$ & 17 & 25,3 \\
\hline & $>20$ & 18 & 26,9 \\
\hline \multirow{3}{*}{$\begin{array}{l}\text { Tiempo de trabajo en el hospital } \\
\text { (horas/semana) }\end{array}$} & $32-47$ & 36 & 53,7 \\
\hline & $48-56$ & 17 & 25,4 \\
\hline & $57-72$ & 14 & 20,9 \\
\hline \multirow{4}{*}{ Años de experiencia } & $1-4$ & 2 & 2,9 \\
\hline & $5-9$ & 2 & 2,9 \\
\hline & $10-19$ & 18 & 26,9 \\
\hline & $>20$ & 45 & 67,2 \\
\hline
\end{tabular}


Como medida de fiabilidad de la encuesta realizada, se calculó el estadístico alfa de Cronbach, el cual arrojó un valor de 0,699 , lo cual se considera adecuado como consenso $(\sim 0,70)$. La encuesta proporciona una calificación global del grado de seguridad del paciente en el servicio/unidad, en una escala de 0 a 10 de mínima a máxima seguridad. Además de que este ítem fue considerado dentro de la dimensión 2 (percepción de la seguridad), es interesante hacer notar que el valor de la mediana fue de 10 , lo cual indica una elevada percepción de la seguridad.

En la figura 1 se pueden observar comparativamente el número de respuestas positivas y negativas en cada dimensión, apreciándose en todos los casos una respuesta mayoritariamente positiva.

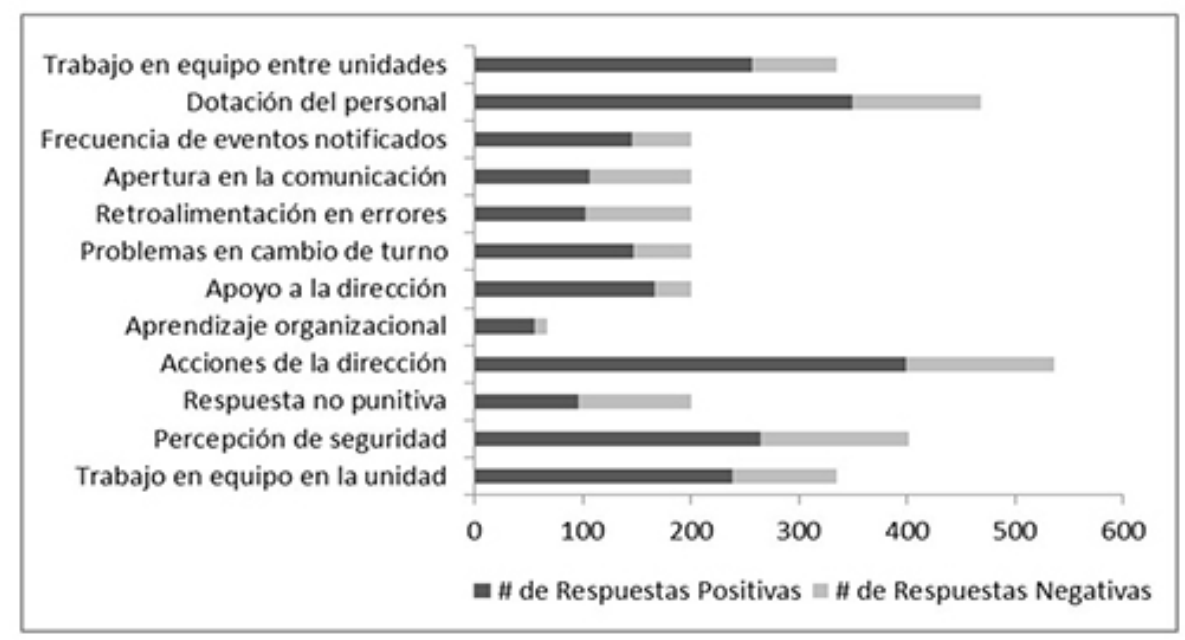

FIGURA 1

Comportamiento de las dimensiones de la cultura de seguridad

Fuente: elaboración propia

Se calcularon los indicadores para cada ítem y dimensión. Se resumen en la tabla 2 los indicadores de cada dimensión en función del porcentaje de respuestas positivas, con sus intervalos de confianza al $95 \%$. Se clasifican como fortaleza las dimensiones: aprendizaje organizacional, apoyo de la dirección y trabajo en equipo entre unidades. El resto de las dimensiones, excepto la respuesta no punitiva (clasificada como debilidad), constituyen oportunidades de mejora. En la dimensión clasificada como debilidad incide fundamentalmente el temor a que quede en el expediente la notificación del error cometido (solo el 26,9\% de los encuestados está de acuerdo con esta acción) (figura 2).

TABLA 2

Indicadores de cada dimensión

\begin{tabular}{llrll}
\hline Dimensión & $\begin{array}{r}\text { Porcentaje de } \\
\text { respuestas } \\
\text { positivas }\end{array}$ & IC 95 \% & Clasificación \\
\hline 1 & Trabajo en equipo en la unidad & 71,3 & $(66,4 ; 76,3)$ & Oportunidad de mejora \\
\hline 2 & Percepción de seguridad & 65,9 & $(61,2 ; 70,7)$ & Oportunidad de mejora \\
\hline 3 & Respuesta no punitiva & 47,8 & $(40,6 ; 54,9)$ & Debilidad \\
\hline 4 & Acciones de la dirección & 74,4 & $(70,6 ; 78,2)$ & Oportunidad de mejora \\
\hline 5 & Aprendizaje organizacional & 82,1 & $(76,2 ; 92,0)$ & Fortaleza \\
\hline 6 & Apoyo a la dirección & 83,1 & $(77,6 ; 88,5)$ & Fortaleza \\
\hline 7 & Problemas en el cambio de turno & 73,1 & $(66,8 ; 79,5)$ & Oportunidad de mejora \\
\hline 8 & Retroalimentación en errores & 51,2 & $(44,1 ; 58,4)$ & Oportunidad de mejora \\
\hline 9 & Apertura en la comunicación & 52,7 & $(45,6 ; 59,9)$ & Oportunidad de mejora \\
\hline 10 & Frecuencia de eventos notificados & 72,6 & $(66,2 ; 79,0)$ & Oportunidad de mejora \\
\hline 11 & Dotación del personal & 74,6 & $(70,6 ; 78,7)$ & Oportunidad de mejora \\
\hline 12 & Trabajo en equipo entre unidades & 76,7 & $(72,0 ; 81,4)$ & Fortaleza \\
\hline
\end{tabular}




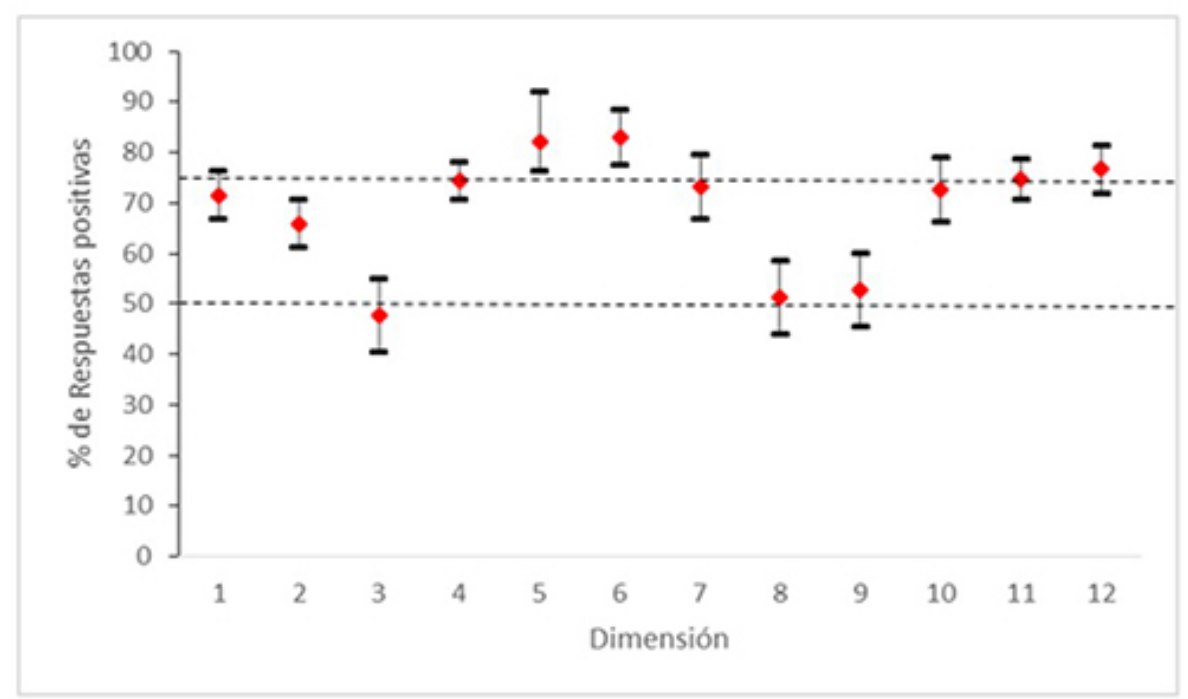

FIGURA 2.

Frecuencia de respuestas positivas

Fuente: elaboración propia

$\mathrm{Al}$ analizar la estimación de los intervalos de confianza y profundizando en los ítems con respuestas positivas inferiores al $75 \%$, se identificaron aquellos en los que se aprecian oportunidades de mejora o debilidades (tabla 3). Como debilidades se identifican: insuficiente personal para afrontar la carga de trabajo, el temor a que quede en el expediente la notificación del error cometido, la vacunación contra la gripe, el temor del personal respecto a hacer preguntas sobre lo que parece incorrecto y que el personal no puede cuestionar con total libertad las decisiones y acciones de sus superiores. Como oportunidades de mejora se debe trabajar en: procurar mayor ayuda entre compañeros, disminuir las jornadas agotadoras, impedir que se produzcan fallos por casualidad, evitar los trabajos bajo presión, no usar los errores como represalia, procurar la búsqueda de las causas de los fallos, no aumentar el ritmo de trabajo si implica sacrificar la seguridad del paciente, intercambiar información, actuar frente a incidentes notificados, informar sobre los errores, expresar con libertad las decisiones o acciones de sus superiores, discutir los errores, prevenir los errores, disminuir errores adversos, anotar en la historia clínica y lograr coordinación entre unidades.

TABLA 3.

Ítems con $<75 \%$ de respuestas positivas

\begin{tabular}{|c|c|c|}
\hline Dimensión & ftem con $<75 \%$ de respuestas positivas & $\begin{array}{r}\text { Porcentaje de } \\
\text { respucstas positivas }\end{array}$ \\
\hline \multirow{2}{*}{ Trabajo en equipo en la unidad } & Suficiente personal pura aftontar la carga de trabajo & 388 \\
\hline & Ayuda cntre companicto & 73.1 \\
\hline \multirow{3}{*}{ Perceppoión de seguridad } & Jotnada sgocadora & 61.2 \\
\hline & No $s$ producen mis fallos por casuulidad & 507 \\
\hline & Trabujo bajo presión & 56,7 \\
\hline \multirow{3}{*}{ Respucsa no punitiva } & $\begin{array}{l}\text { Si los companieros o superiores se enteran de que has cometido un error lo } \\
\text { usan en tu contra }\end{array}$ & 64.2 \\
\hline & Cuando se detecta un fallo, antes de buscat la causs s busca un culpuble & 52.2 \\
\hline & Cunndo se comete un errot, el personal teme que quede en su expediente & 269 \\
\hline \multirow{4}{*}{$\begin{array}{l}\text { Aeciones de la difoceión que } \\
\text { favorecen la scguridad } \\
\text { Problemas en cambio de turno }\end{array}$} & Vacunación contra la gripe & 28,4 \\
\hline & Ritmo de trabujo & 56,7 \\
\hline & Intercambio de información & 64,2 \\
\hline & Actwaciones ante incidentes notificados & 61.2 \\
\hline \multirow[t]{2}{*}{ Retroulimentación en ctroess } & Se informan los errores & 62.7 \\
\hline & El personal teme hacer preguntas sobre lo que parece incortecto & 299 \\
\hline \multirow{3}{*}{ Apcrtura en la comunicasión } & $\begin{array}{l}\text { Cunndoel personal ve algo que puede afectar nogutivamente a la atención } \\
\text { del paciente habla de ello con toeal libertad }\end{array}$ & 64.2 \\
\hline & $\begin{array}{l}\text { El peronal puede cuestionar con total libertad las decisiones u acciones de } \\
\text { sass superiores }\end{array}$ & 269 \\
\hline & Discusión de ertores & 67,2 \\
\hline \multirow{2}{*}{ Frecuencia de eventos notificados } & Prevención de crtores & 68.7 \\
\hline & Errores adverios & 73.1 \\
\hline \multirow[t]{2}{*}{ Dotación del personal } & $\begin{array}{l}\text { Anotación en la historia dínica sobre tratamientos, cuidadoso } \\
\text { proxedimientos }\end{array}$ & 500 \\
\hline & Revisión de historia clinica & 70,1 \\
\hline Trabajo en equipo entre unidades & Coordinación entere unidades & 67.2 \\
\hline
\end{tabular}

Fuente: elaboración propia 


\section{Discusión}

En relación con las fortalezas identificadas en este estudio, como son aprendizaje organizacional, se encontró similitud a lo reportado en los trabajos de Wang y Tao (4), los de Hernández Montes et al. (7), Meléndez Méndez et al. (9) y Castañeda et al. (12), y la dimensión "trabajo en equipo entre unidades" se encontró similitud con los estudios de Khater et al. (2), Wang y Tao (4) y Castañeda et al. (12).

En cuanto a las dimensiones de oportunidades de mejora o debilidades, se destaca la respuesta no punitiva a los errores, similar al estudio de Wang y Tao (4), la retroalimentación en errores y la apertura en la comunicación similar al estudio de Orkaizagirre Gómara (13).

La vivencia de los encuestados frente a los errores que cometen está matizada por el temor a las represalias y a que quede reflejado en su expediente, lo que lleva a un subregistro de errores cometidos, sin importar si son potencialmente dañinos o no. Es necesario consolidar una cultura institucional educativa y no punitiva que parta del informe y análisis de los errores y eventos adversos, en la que se reconozca que los errores siempre van a existir, porque hacen parte de los riesgos cuando se presta atención en salud y que el sistema de reporte de eventos adversos es un mecanismo que ayuda a los profesionales de la salud a minimizarlos, con similitud con los trabajos de Hernández Montes et al. (7), Meléndez Méndez et al. (9) y Pozo Muñoz y Padilla Marín (14).

En cuanto a la dimensión de la cultura, que hace referencia a la composición de la fuerza de trabajo, se percibe que falta un mayor número de trabajadores para prestar una atención adecuada a los usuarios, lo cual podría generar recarga y aumento en la posibilidad de error, por apresuramiento o por cansancio. Esto es similar a los estudios de Bernalte Martí et al. (6) y Hernández Montes et al. (7). Este aspecto tiene que ver con uno de los tres componentes clave de la calidad en la prestación de servicios de salud: contar con personal en número suficiente para atender eficazmente las demandas de atención, lo cual es clave para apoyar la implementación de la cultura de seguridad del paciente.

El manejo de la comunicación es otra dimensión clave de la cultura de seguridad del paciente en el quirófano. Se evidencia gran necesidad de mejoramiento en este aspecto de la cultura, similar al estudio de Meléndez Méndez et al. (9).

En síntesis, es necesario consolidar el uso del sistema de reportes de eventos adversos y la compresión de su utilidad en el quirófano, trabajando para que el error sea asumido como un riesgo en la prestación del servicio y no como un problema ético o de ineficiencia técnica.

En la nueva comprensión del tema se requiere trabajar para que la gente comprenda que el reporte podría mejorar la calidad y la seguridad del paciente, al tiempo que se garantiza que no habrá represalias y que todos conocen el sistema (2,4-9). Es necesario remplazar la cultura tradicional de la vergüenza/culpa con una cultura no punitiva (15).

Una de las limitaciones que tiene este estudio es que solamente se ha centrado en el proceso de seguridad quirúrgica en una muestra pequeña. El avance en esta estrategia de seguridad en todas las unidades hospitalarias de atención quirúrgica del país permitirá conocer el cambio de cultura de las organizaciones de salud en esta materia.

\section{Conclusiones}

El personal de enfermería que labora en estas unidades quirúrgicas percibe un buen clima de seguridad. Las fortalezas identificadas por los enfermeros hacia la cultura de seguridad fueron las dimensiones aprendizaje organizacional, apoyo a la dirección y trabajo en equipo entre unidades.

Las dimensiones percibidas hacia la mejora continua fueron: trabajo en equipo en la unidad, percepción de seguridad, acciones de la dirección, problemas en el cambio de turno, retroalimentación en errores, apertura 
en la comunicación, frecuencia de eventos notificados y dotación del personal. La dimensión respuesta no punitiva a los errores se percibe como una debilidad.

\section{Referencias}

1. Columbié Pileta M, Morasen Robles E, Moya Bisset Y. Cultura en seguridad del paciente en estudiantes de FATESA. Rev Cub Tec Sal [internet]. 2016 [citado 2017 Abr 25];7(2):28-37. Disponible en: https://www.medigraphic. $\mathrm{com} /$ cgi-bin/new/resumen.cgi?IDARTICULO=68976

2. Khater WA, Akhu-Zaheya LM, Al-Mahasneh SI, Khater R. Nurses' perceptions of patient safety culture in Jordanian hospitals. Rev Int Nurs. 2015;62(1):82-91. https://doi.org/10.1111/inr.12155

3. Torijano Casalengua M, Olivera Cañadas G, Astier Peña MP, Maderuelo Fernández JA, Silvestre Busto C. Validación de un cuestionario para evaluar la cultura de seguridad del paciente de los profesionales de atención primaria en España. Atención Primaria. 2013 Ene;45(1):21-37. https://doi.org/10.1016/j.aprim.2012.07.003

4. Wang M, Tao H. How does patient safety culture in the surgical departments compare to the rest of the county hospitals in Xiaogan city of China? Int J Environ Res Public Health. 2017 Sep;14(10). https://doi.org/10.339 0/ijerph14101123

5. Akbari N, Malek M, Ebrahimi P, Haghani H, Aazami S. Safety culture in the maternity unit of hospitals in Ilam province, Iran: a census survey using HSOPSC tool. Pan Afr Med J. 2017 Ago;27:268. https://doi.org/10.116 04/pamj.2017.27.268.9776

6. Bernalte Martí V, Orts-Cortés MI, Maciá-Soler L. Percepción de los profesionales de enfermería y auxiliares de enfermería sobre cultura de seguridad del paciente en el área quirúrgica. Enferm Clín. 2015;25(2):64-72. https ://doi.org/10.1016/j.enfcli.2014.08.002

7. 7. Hernández-Montes YM, Valle-Alonso J, García-Jiménez CM, Lopera-Lopera E, Luna-Chamizo R, FernándezBejarano JA. Cultura de seguridad del paciente en el servicio de urgencias del Sistema Andaluz de Salud. Revista CONAMED [internet]. 2013 [citado 2016 abr 2];18(4):148-56. Disponible en: https://dialnet.unirioja.es/se rvlet/articulo?codigo $=4701301$

8. Meléndez Méndez C, Garza Hernández R, Castañeda-Hidalgo H, González Salinas J, Turrubiates Pérez J. Percepción del paciente quirúrgico acerca de la seguridad en el ámbito hospitalario. Rev Cuid. 2015;6(2):1054-61. http://dx.doi.org/10.15649/cuidarte.v6i2.165

9. Meléndez Méndez C, Garza Hernández R, Salinas J, Castillo Castillo S, González García S, Ruiz Hernández E. Percepción del personal de enfermería hacia la cultura de seguridad en un hospital pediátrico en México. Rev Cuid. 2014;5(2):774-81. http://dx.doi.org/10.15649/cuidarte.v5i2.92

10. Feldt LS, Woodruff DJ, Salih FA. Statistical inference for coefficient alpha. Appl Psychol Meas. 1987;11:93-103. https://doi.org/10.1177/014662168701100107

11. Cuestionario sobre seguridad de los pacientes: versión española del Hospital Survey on Patient Safety [internet]. Madrid: Ministerio de Sanidad y Consumo; 2005. Disponible en: https://www.mscbs.gob.es/organizacion/sns /planCalidadSNS/docs/CuestionarioSeguridadPacientes1.pdf

12. Castañeda Hidalgo H, Garza Hernández R, González Salinas JF, Pineda Zúñiga M, Acevedo Porras G, Aguilera Pérez A. Percepción de la cultura de la seguridad de los pacientes por personal de enfermería. Rev Cienc Enferm. 2013;19(2):77-88. http://dx.doi.org/10.4067/S0717-95532013000200008

13. Orkaizagirre Gómara A. La cultura de seguridad del paciente de las enfermeras hospitalarias [tesis doctoral] [internet]. Zaragoza: Universidad de Zaragoza, Fisiatría y Enfermería; 2016. Disponible en: https://zaguan.un izar.es/record/48392/files/TESIS-2016-139.pdf

14. Pozo Muñoz F, Padilla Marín V. [Assessment of the patient-safety culture in a healthcare district]. Rev Calid Asist. 2013;28(6):329-36. https://doi.org/10.1016/j.cali.2013.03.009

15. Tereanu C, Sampietro G, Sarnataro F, Siscanu D, Palaria R, Savin V et al. Survey on patient safety culture in the Republic of Moldova: a baseline study in three healthcare settings. Clujul Med. 2018;91(1):65-74. https://doi .org/10.15386/cjmed-869 


\section{Notas}

* Artículo original de investigación

Conflictos de interés: la autora declara no tener ningún conflicto de interés.

Financiamiento: ninguno

\section{Licencia Creative Commons CC BY 4.0}

Cómo citar este artículo: Pérez Bolaños L. Percepción de enfermería. Una mirada actual a la cultura de seguridad en el quirófano. Investig Enferm Imagen Desarr. 2020;22. https://doi.org/10.11144/ Javeriana.ie 22.pem 